\title{
Mark BAILONI, La question régionale en Angleterre: nouvelles approches politique du territoire anglais
} Thèse de Doctorat, Université Paris 8, Institut Français de Géopolitique, 2007

\section{Simon Edelblutte}

\section{OpenEdition}

\section{Journals}

Édition électronique

URL : http://journals.openedition.org/rge/1102

DOI : $10.4000 /$ rge. 1102

ISSN : 2108-6478

Éditeur

Association des géographes de l'Est

Édition imprimée

Date de publication : 1 janvier 2008

ISSN : 0035-3213

Référence électronique

Simon Edelblutte, «Mark BAILoNı, La question régionale en Angleterre : nouvelles approches politique du territoire anglais », Revue Géographique de l'Est [En ligne], vol. 48 / 1-2 | 2008, mis en ligne le 15

décembre 2009, consulté le 22 septembre 2020. URL : http://journals.openedition.org/rge/1102 ; DOI https://doi.org/10.4000/rge.1102

Ce document a été généré automatiquement le 22 septembre 2020.

Tous droits réservés 


\title{
Mark BAILONI, La question régionale en Angleterre: nouvelles approches politique du territoire anglais
}

Thèse de Doctorat, Université Paris 8, Institut Français de Géopolitique, 2007

\author{
Simon Edelblutte
}

\section{RÉFÉRENCE}

Mark BAILONI, La question régionale en Angleterre : nouvelles approches politique du territoire anglais, Thèse de Doctorat, Université Paris 8, Institut Français de Géopolitique, 2007, $560 \mathrm{p}$.

En une thèse de 560 p., M. Bailoni traite de la question régionale en Angleterre. Il s'agit d'un travail d'analyse géographique associé à une démarche géopolitique (p. 11 à 13). C'est-à-dire que l'auteur tient d'abord aux spécificités de la géographie, par l'observation et l'analyse des territoires pour en comprendre les problèmes et en mesurer les défis locaux, régionaux et nationaux (p. 11). La cartographie a d'ailleurs un place très importante dans le volume, avec des cartes efficaces sur le fond et lumineuses sur la forme (p. 273, 436, 437, 450, 479...), idéales pour expliquer les nombreux enjeux qui affectent ces territoires à différentes échelles. L'auteur analyse ainsi efficacement les rivalités de pouvoirs sur des territoires, le jeu des acteurs et les représentations des populations; c'est en cela qu'il s'agit aussi d'une démarche géopolitique.

\section{Le sujet et la démarche}

2 Cette question est d'actualité dans un État-multination, longtemps considéré comme le pays le plus centralisé d'Europe. En effet, Il a été exclusivement ou presque gouverné 
par Londres durant tout le $\mathrm{xx}^{\mathrm{e}}$ siècle. L'auteur en donne la raison essentielle dès l'introduction, en évoquant l'opposition entre, d'une part, des nations périphériques faibles démographiquement et économiquement mais à l'identité affirmée, et, d'autre part, une nation centrale puissante et peuplée qui a la taille d'un État majeur à l'échelle européenne (50 millions d'habitants) : l'Angleterre.

3 Puis, en 1997, avec l'arrivée au pouvoir des néo-travaillistes, les «nations périphériques" du Royaume-Uni, Écosse et Pays de Galles, ont bénéficié de la dévolution, c'est-à-dire du transfert de pouvoirs importants de Londres vers Edinburgh ou Cardiff. Plus tard, l'Irlande du Nord a retrouvé une autonomie déjà acquise mais suspendue quelques décennies ou pendant les «troubles", et Londres a obtenu un maire élu. Ainsi, le Royaume-Uni, État unifié, mais pas État uniforme (p. 6), est un pays où "l'approche de la question régionale [...] est beaucoup plus complexe que dans le Étatsnations unitaires » (p. 6) tels que la France.

4 Cette question régionale, qui concerne des territoires politiques intermédiaires entre le niveau local et le niveau national, est extrêmement complexe et particulière au Royaume-Uni, du fait du profond déséquilibre entre ses nations constituantes. Si l'Ecosse, le Pays de Galles et l'Irlande du Nord ont une taille régionale, l'Angleterre ne peut être considérée comme une région en raison de sa taille et surtout de sa population. Ainsi la question régionale existe également à un niveau inférieur, c'est-àdire à l'intérieur d'un territoire qui n'est pas un État, l'Angleterre. Le problème est donc, dans un souci d'efficacité de gestion, mais aussi de rapprochement du pouvoir et du citoyen - Londres est loin vue de Liverpool ou de Newcastle - d'inventer de nouveaux territoires et de leur donner de nouvelles compétences, suivant ainsi une tendance de fond dans l'Union Européenne (UE) depuis plusieurs décennies. En bref, il s'agit de donner un poids politique réel à des régions anglaises sans pouvoirs élus face aux puissants Länder allemands ou même face aux régions françaises, qui disposent déjà d'une autonomie certaine.

5 Pour réussir un tel travail de recherche, l'auteur s'est évidement appuyé sur des ressources bibliographiques, mais aussi et surtout sur le dépouillement systématique des grands journaux britanniques. Cela a été rendu nécessaire par l'évolution rapide du sujet traité, notamment au moment de la préparation et du bilan du référendum de novembre 2004 sur la dévolution dans le Nord-Est. Mais l'intérêt du travail réside aussi dans la quarantaine d'entretiens (liste p. 527-528) réalisés lors de fréquents séjours en Angleterre (dont un séjour d'une année à l'université de Newcastle) avec «des élus, des chercheurs, des agents de développement, des personnalités politiques, des lobbyistes ou fonctionnaires» (p. 16). Ces entretiens, en plus de permettre un travail sur la perception de cette question régionale, apportent aussi une dimension humaine certaine à un ouvrage ancré sur le terrain tout en complétant heureusement les réflexions plus conceptuelles.

\section{Un emboîtement spatial et chronologique}

Le volume s'articule en trois parties organisées en une sorte d'emboîtement spatial et chronologique, partant du Nord-Est et de son référendum pour évoquer la lente régionalisation de l'Angleterre et le développement assez récent des identités régionales. 
7 La première partie est essentiellement consacrée à la grande échelle et à l'actualité brûlante de la région Nord-Est de l'Angleterre. En effet, le référendum organisé dans cette région en novembre 2004 et qui devait entériner la politique du New Labour en étendant la dévolution écossaise et galloise (dans une moindre mesure cependant) au Nord-Est de l'Angleterre, a été un échec retentissant pour le pouvoir. Le résultat, un «non » à 78 \%, était inattendu puisque encore 2 semaines avant le scrutin (p. 50), les sondages annonçaient, dans la lignée d'un mouvement non seulement national mais aussi européen, une victoire $\mathrm{du}$ « oui ». M. Bailoni interprète ce résultat comme non pas un rejet réel d'une dévolution qui ne passionnait certes pas les électeurs du Nord-Est tant elle était timide, mais comme un vote «d'une portée beaucoup plus générale dans le contexte politique britannique du moment» (p.22). En effet, la région est un bastion travailliste depuis très longtemps et le rejet d'une proposition d'un gouvernement travailliste y a évidement plus de poids qu'ailleurs.

Replaçant ensuite le vote dans le contexte national de la dévolution «à géométrie variable " pratiquée par T. Blair en donnant des pouvoirs différents à des moments différents et à des régions différentes, l'auteur décrit ensuite le fameux projet, jugé comme trop peu ambitieux. Il évoque les campagnes différentes et passionnées qui se sont affrontées, pointant le rôle parfois trouble d'élus travaillistes craignant pour leur mandat et leur pouvoir si la mise en place d'une assemblée régionale était votée. Si le «non» peut en partie être expliqué par le contexte politique international et notamment le mécontentement d'une grande majorité d'Anglais (en particulier travaillistes) face au soutien de T. Blair à l'intervention militaire américaine en Irak, il est surtout dû à une erreur de calendrier (p. 88 à 94). Le vote est en effet jugé d'abord trop tardif par rapport aux dévolutions écossaises et galloises, dont certains aspects négatifs - notamment financiers - apparaissent alors dans les médias. Il est aussi trop proche d'élections générales (2005), donnant l'occasion inespérée à des électeurs très majoritairement travaillistes de donner un coup de semonce à un gouvernement de même bord sans pour autant risquer un retour des conservateurs au pouvoir (p. 94).

9 L'auteur tire ensuite les leçons politiques de ce référendum raté, avec des conséquences variées sur une classe politique globalement affaiblie. Le parti travailliste, s'il est en recul au niveau régional, notamment en raison d'un redécoupage électoral peu favorable, ne cède cependant rien aux Conservateurs, très discrédités depuis les années Thatcher dans une région frappée par une crise industrielle profonde et précoce. Par contre, au-delà des petits partis populistes, anti-européens et plus ou moins sérieux (British National Party, United Kingdom Independance Party, ou encore Neil Herron, président de l'association des martyrs du système métrique), c'est le parti LibéralDémocrate (Lib-

Dem) qui profite, tant lors des scrutins locaux que nationaux, du recul des travaillistes. Le basculement dans le camp Lib-Dem de Newcastle, ville qui a connu ces dernières années un profond renouveau urbain accompagné d'un changement de la composition socio-politique de la population, en est le symbole le plus fort.

10 Ainsi, le référendum raté du Nord-Est révèle-t-il un climat anti-labour et antiestablishment, tout en illustrant le mécontentement et le désenchantement des électeurs vis-à-vis de leurs élus et de l'ensemble de classe politique ; il peut en cela être comparé au "non » français à la Constitution européenne en mai 2005 (p. 156). Plus grave, ce «non » ralentit la réforme administrative et politique de l'Angleterre tout en accentuant le déséquilibre institutionnel du Royaume-Uni qui juxtapose donc des 
périphéries à forte identité et assez autonomes, un centre londonien tout puisant et une Angleterre provinciale sans pouvoir. Le résultat est d'autant plus étonnant que, s'il n'y a pas de pouvoir régional en Angleterre, le territoire anglais est depuis longtemps administré par le pouvoir central sur des bases régionales; c'est l'objet de la seconde partie.

11 La seconde partie traite essentiellement des découpages régionaux de l'Angleterre, mis en place dès les années 1920 pour pallier les difficultés des territoires en crise. La partie commence par une mise au point sur le contexte européen, puis brosse un portrait des institutions régionales en Angleterre, toutes dessinées ou tout au moins contrôlées par l'État central : Government Offices for Regions (GOR), Regional Development Agencies (RDA), Regional Chambers ou encore Quasi Autonomous Non Governmental Organisations (quangos), "agences de l'État chargées d'appliquer les décisions de leur ministère de tutelle au niveau territorial sur lequel elles s'exercent » (p. 186). Ce dernier terme est considéré comme péjoratif et sous-entend qu'un pouvoir qui devrait être aux mains d'élus du peuple, est exercé par des agents souvent nommés et donc sans réelle représentativité démocratique. Le terme "quangos» rassemble donc une multitude d'agences ou d'organismes très différents, qui travaillent souvent de manière très autonome (p.187). Toutes ces institutions restent finalement en place puisque l'assemblée régionale a été refusée dans le Nord-Est.

On peut cependant regretter ici une partie très technique, donc quelque peu indigeste et qui aurait mérité quelques schémas explicatifs, mais elle permet néanmoins d'amorcer l'historique beaucoup plus intéressante de la régionalisation de l'Angleterre par des découpages venus du gouvernement central, différent en cela d'un régionalisme qui viendrait des habitants et des élus locaux. La série de cartes des pages 204 à 223 montre la taille de plus en plus grande de territoires aidés (assisted areas) par le gouvernement central, puis leur rétractation sous les gouvernements conservateurs de Thatcher et Major, qui réduisent également les pouvoirs des autorités locales en redécoupant les territoires politiques locaux. C'est néanmoins John Major qui crée les GOR pour « organiser, coordonner et modéliser les politiques régionales au nom de la rationalité et de l'efficacité» (p.226) amorçant une régionalisation administrative plus complète et autorisant ainsi une régionalisation politique (dévolution) que les Travaillistes, alors dans l'opposition, réclamaient.

13 Arrivés au pouvoir en 1997, le New Labour, au-delà des emblématiques dévolutions écossaises et galloises, développe une nouvelle approche territoriale, renforçant le rôle des institutions régionales et laissant de plus en plus de place aux initiatives locales, notamment dans le domaine du renouveau urbain (Urban Development Corporation, Urban Regeneration Companies, et du rattrapage du retard des régions du Nord par rapport au cœur londonien (Northern way, City Regions).

14 Enfin, la dernière sous-partie, passionnante, pose la question des limites régionales de l'Angleterre en présentant un série de cartes des découpages anciens (comtés historiques) et des découpages régionaux proposés au cours $d u x^{e}$ siècle. L'auteur $\mathrm{y}$ montre ainsi la lente maturation des Standard Regions, découpage actuel qui s'amorce au début des années 1970. Ces limites - comme toute limite - sont néanmoins contestées, notamment autour de Londres où l'influence de la capitale s'exerce largement et directement sur les régions East of England et South East, et dans le Nord, sorte d'archipel urbain où aucune des anciennes villes industrielles (Manchester, Liverpool, Leeds, Sheffield, Bradford, Newcastle, Sunderland...) ne polarise ni vraiment 
ni clairement un large territoire, ce qui pose donc d'ailleurs la question des capitales régionales.

Enfin, la troisième partie traite, suite à l'échec du référendum, des nouvelles approches pour la dévolution en Angleterre. Les dévolutions écossaise et galloise ont en effet provoqué, en contrecoup, un regain de l'identité anglaise face à l'identité britannique en même temps que de nouvelles tensions entre les Nations composantes du RoyaumeUni, allant parfois jusqu'à poser la question de sa survie. "West Lothian question » (du nom de la circonscription d'un député hostile au projet avorté de dévolution de 1978) qui pose le problème de la compétence non réciproque des députés écossais sur les affaires anglaises ; formule Barnett, système de répartition des ressources de l'État très avantageux pour l'Écosse et le Pays de Galles, sont autant de problèmes qui posent plus généralement la question de la très difficile distinction entre les affaires anglaises et les affaires britanniques (p. 337).

La situation est encore compliquée par l'évolution ethnique et culturelle du RoyaumeUni où les rapports de force entre les veilles nations écossaise, galloise, irlandaise et anglaise ont été bouleversés, non seulement par les changements économiques profonds, avec la crise industrielle, mais aussi par l'arrivée des minorités ethniques (les immigrés caribéens, africains ou asiatiques et leurs descendants) qui se sentent britanniques avant tout et ne s'identifient pas à l'une ou l'autre des nations anciennes du Royaume-Uni.

$17 \mathrm{Au}$ cœur de cette évolution apparaît, en réaction aux affirmations écossaises et galloises, un développement certain de l'anglicité. Multiplication des croix de SaintGeorges à la place des Union Jack lors des compétitions sportives; commémoration toujours plus importante de la Saint-Georges, sont autant de manifestations de cette anglicité qui a néanmoins encore des difficultés à bien se distinguer de la britannicité, en raison de l'héritage politique et institutionnel ou de la suprématie économique et démographique de l'Angleterre. Le développement de petits partis europhobes et populistes (comme l'English Democrats Party) dont le discours défendant les parties riches du territoire face aux parties pauvres, se rapproche de celui du Vlams Belang flamand en Belgique et de la Ligue du Nord en Italie (p.377), reste cependant anecdotique et ne devrait pas aboutir à court ou à moyen terme, à la création d'un parlement anglais. Cette constatation s'appuie aussi sur le fait que les fractures internes à l'Angleterre elle-même sont importantes.

18 La sous-partie 3.2 traite de ces inégalités spatiales; c'est peut-être la plus intéressante et la plus originale de cette thèse. L'auteur y montre en en effet le développement de l'idée régionale, d'ailleurs souvent portée par des géographes, dans le Nord et le NordEst de l'Angleterre. Face à la politique thatchérienne d'abandon de la veille industrie, un sentiment d'injustice s'est construit autour de la fracture Nord-Sud (selon une ligne The Wash/Bristol), dont les origines remonteraient, selon certains, à la géographie physique (massifs anciens au Nord et bassin sédimentaire au Sud). Si les différents indicateurs montrent effectivement, à l'échelle nationale, une richesse et un développement plus fort dans le Sud-Est que dans le Nord, M. Bailoni croise intelligemment les différentes échelles régionales et locales par une série de cartes (p. 421 à 427 et p. 436 et 437) qui nuancent cette division abondamment reprise par les médias et les tenants de l'autonomie du Nord. Le clivages intra-régionaux et plus encore entre la ville et la campagne sont tout aussi saisissants et affectent le Sud-Est et 
Londres comme les régions du Nord; ils sont particulièrement visibles dans le domaine électoral. fonde donc autant sur des représentations que sur la réalité. L'image magnifiée de la vieille industrie, dont la destruction n'est due, selon les régionalistes, qu'à la politique du Sud, donc de Londres, est au cœur de ces représentations, accompagnée par les particularismes historiques, culturels et linguistiques du Nord-Est, très bien repris sur la carte de la page p. 450.

L'auteur traite ensuite plus rapidement des autres identités régionales en Angleterre (Cornouailles, voire Sud-Est), brillamment résumés par l'excellente carte de la p. 479. Il termine enfin par une sous-partie traitant des volontés de réforme du gouvernement local. Devraient ainsi s'affirmer, dans un objectif de rapprochement des citoyens et du pouvoir, des territoires politiques plus petits («constituencies» de Birmingham) et inférieurs aux autorités unitaires, en même temps que devaient être supprimés les doublons comtés/districts par la généralisation des autorités unitaires. Parallèlement, de nouvelles pistes, comme les City Regions, nouveaux territoires développées autour des plus grandes villes et de leur espace polarisé, sont explorées.

\section{Un apport essentiel sur le Royaume Uni}

L'ouvrage permet donc au lecteur intéressé par le Royaume-Uni et par toutes les problématiques de la régionalisation et du régionalisme, de faire le point sur l'évolution de la situation dans un État qui apparaît peu à peu, au fil de la lecture du volume, comme un terrain d'expériences territoriales à la fois novatrices et respectueuses de la tradition (certaines limites de districts ou de comtés n'ont pas évolué depuis le Domesday Book de 1086 : carte p. 306). Cet apparent paradoxe donne des découpages régionaux très différents du système français, très cartésien dans son emboîtement et dans son uniformité presque totale. M. Bailoni montre ainsi que l'originalité des réponses anglaises aux difficultés des découpages régional et local réside dans leur variété, puisque tous les citoyens ne sont pas traités de la même façon par l'administration territoriale. Qu'ils soient écossais bénéficiant de la dévolution, Londoniens disposant d'un maire élu, habitant du Nord-Est ayant refusé la dévolution, les Britanniques sont incontestablement entrés, depuis la dévolution de 1997, dans une phase intense de recomposition de leurs territoires politiques infra-étatiques qui, par certains côtés, répond à l'intégration du pays dans l'ensemble supra-étatique qu'est l'Union Européenne.

La thèse a été soutenue à l'université Paris 8 le 10 décembre 2007 et a obtenu la mention très honorable avec les félicitations du jury. 


\section{AUTEURS}

\section{SIMON EDELBLUTTE}

CERPA, Nancy Université 